This is corroborated by the atomic heat of formation of selenium dioxide, which has been calculated as $\mathrm{Se}+\mathrm{O}+\mathrm{O}=\mathrm{SO}_{2}+8 \cdot 9$ volts, which is almost double the energy of dissociation of $\mathrm{SeO}$.

\author{
Dept. of Physics, \\ Muslim University, \\ Aligarh. \\ Sept. 1. \\ ${ }^{1}$ R. K. Asundi and R. Samuel, Proc. Ind. Ac. Soc. (Bangalore), 2, \\ $30 ; 1935$. \\ G. Herzberg and E. Teller, Z. Phys. Chem., B, 21, 410; 1933.
}

\section{The L-Emission Spectrum of Argon}

OF the X-spectra of rare gases, the $K$-series of xenon and that of krypton have been measured ${ }^{1}$, and from their absorption spectra the $K$-edge of argon $^{2}$, krypton ${ }^{3}$ and the $L$-edge of xenon ${ }^{3}$ are known. For the $L$-absorption edge of $\mathrm{Xe}$, as well as for the $K$-edge of argon ${ }^{4}$, a fine structure also has been found.

In the region of the long wave-lengths, no results obtained by direct measurements have been obtained hitherto. The values for absorption edges have been obtained by the method of magnetic spectra.

By studying with our ionic tube the reversed absorption edges of silver, bromine and potassium in the region of long wave-lengths ${ }^{5}$ (which we will publish later on), we have found that if either $\mathrm{N}_{2}$ or air has been let into the tube, the $K$-series of $\mathrm{N}_{2}$ has occurred. Sometimes, there also appeared the absorption edge of nitrogen ${ }^{6}$. The absorption edge of $\mathrm{N}_{2}$ appeared especially when the same pressure had been maintained in the spectrograph as in the ionic tube. Such a regulation and maintenance of the pressure in the spectrograph-which is possible by the application of the ionic tube-is important in the region of the long wave-lengths, where it is impossible to cover the slit. (The gas, which is let into the tube by means of a fine regulating glass valve? $^{\text {, }}$ streams through the slit into the spectrograph, from which it is exhausted.) We have tried to obtain spectra of the rare gases mentioned with the arrangement described above, where the said ionic tube offers a very efficient source of the long $\mathrm{X}$-rays ${ }^{8}$. It is now possible to operate with this tube with a tension of only $600 \mathrm{v}$. at $250-300 \mathrm{ma}$. The pressure in the tube under the given conditions is about $0 \cdot 1 \mathrm{~mm}$. $\mathrm{Hg}$.

When argon is let in the above-mentioned way into the tube, the $L$-series of argon is obtained. As electrodes we have used aluminium with the deposit of lithium borate on the anticathode. For control, if $\mathrm{H}_{2}$ has been let in instead of argon, only $K$-lines of boron (in 8 orders), $K$-lines of lithium and the $K$-absorption edge of carbon in the first and the second orders have been obtained. But when argon is let in, besides the expected argon lines, we have obtained also new lines, which are observed when $\mathrm{H}_{2}$ has been the discharge gas. These new lines have been found to belong simply to the elements calcium, sodium and silicon, which elements have been sputtered on the anticathode from some parts of the tube by the presence of argon. The $L$-series of argon have been measured with reference to the lines of boron $^{9}$, and the following values have been obtained

$\begin{array}{lccr} & \lambda & \nu / R & \nu / R \text { cal. } \\ L_{e} & 56 \cdot 1 & 16 \cdot 2 & 16 \cdot 29 \\ L_{\eta} & 56 \cdot 7 & 16 \cdot 1 & 16 \cdot 13\end{array}$

The observed values (measured in the second order) agree well with those calculated by interpolation of the data from Siegbahn and Magnusson ${ }^{10}$. We have not succeeded hitherto in obtaining a distinct character of the absorption edge of argon. The short wave limit of these lines is $54.0 \mathrm{~A}$., which differs from the value given by Holweck $(50 \cdot 1 \mathrm{~A}$.) and by Turner $(50.7 \mathrm{~A}$.) by about $3 \mathrm{~A}$. Our results show that, with certain modifications of our arrangement, such a pressure is obtainable that we can expect the above mentioned absorption edges of argon, as well as of other gases, in the region of long wave-lengths.

M. BAČKovskŕ

V. DOLEJŠEK.

Spectroscopical Institute

of the Charles University, Prague.

Sept. 16.

\author{
1 A. Dauvillier, C.R., Paris, 191, 937 ; 1930. \\ 2 H. Fricke, Phys. Rev., 15, 202; 1920 . \\ Y. D. Hanawalt, Phys. Rev., 37, 715; 1931 \\ D. Coster and Van der Tuuk, Z. Phy8., 37, 367 ; 1926. Nature, \\ 117, $586 ; 1926$. \\ , Y ; 1926 . nd V. Kunzl, C. Y. M. Fys , 61, 242; 1939. Z. Phys. \\ 74. $565 ; 1932$. \\ J. Thibaud, J. Phys. et Rad., 8, 447 ; 1927. \\ 7 V. Kunzl and J. Slavik, Z. Techn. Phys., August, 1935 \\ V. Kunzl, NATURE, 136, 437 ; 1935 \\ M. Siegbahn and T. Magnusson, II, Z. Phys., 87, 305; 1935. \\ ${ }^{10} \mathrm{M}$. Siegbahn and T. Magnusson, IV, Z. Phys., 95, 149 ; 1935.
}

\section{Dipole Moments of Ethyl and Iso-amyl Borates and Triphenyl Phosphate}

Some months ago we commenced an examination of the dipole moments of esters of inorganic acids.

Otto $^{1}$ has now published the dipole moments of $n$-butyl borate and $n$-amyl borate as $0.77 \mathrm{D}$ and $0.79 \mathrm{D}$, respectively, at $25^{\circ}$, in benzene. We have found that ethyl and $i s o$-amyl borates have moments of $0.75 \mathrm{D}$ and $0.81 \mathrm{D}$, respectively, at $20^{\circ}$. The values for $n$ - and iso-amyl borates are, therefore, in good agreement, and there seems to be a slight increase in the moment in ascending the series of alkyl groups. Triphenyl phosphate has, we find, a moment of $2 \cdot 79 \mathrm{D}$.

The borates were fractionated several times, and the triphenyl phosphate recrystallised twice from petrol ether. As the borates are extremely easily hydrolysed, great care was necessary to avoid exposure to air. The dielectric constants were measured by the apparatus previously used ${ }^{2}$, except that a glass dielectric cell containing platinum plates, the inlet and outlet tubes of the cell being fitted with ground glass caps, was employed. Benzene was the solvent and all the measurements were at $20^{\circ} \mathrm{C}$.

The results were :

\begin{tabular}{lccc}
\multicolumn{1}{c}{ Compound } & B. Pt. & $d_{\mathbf{s}^{2}}$ & $n_{D}^{20}$ \\
Ethyl Borate & $119 \cdot 2^{\circ} / 756 \mathrm{~mm}$. & 0.8635 & 1.3741 \\
Iso-amyl Borate & $256 \cdot 1^{\circ} / 761 \mathrm{~mm}$. & 0.8514 & 1.4156 \\
Triphenyl phosphate & $49 \cdot 8^{\circ}$ (M.Pt.) & &
\end{tabular}

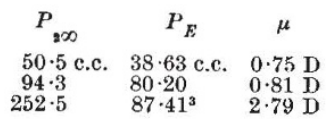

Work is in progress on other esters, and the results will be discussed when the measurements have been completed.

Queen Mary College,

University of London.

E. G. Cowley.

J. R. Partington. Sept. 13.

1 Otto, J. Amer. Chem. Soc., 57, 1477 ; 1935.

2 Cowley and Partington, J. Chem. Soc., 604; 1935.

3 Value calculated from sum of atomic refractions for D line. 